\title{
VARIABILITY IN COBB ANGLE MEASUREMENTS IN CHILDREN WITH CONGENITAL SCOLIOSIS
}

\author{
RANDALL T. LODER, ANDREW URQUHART, HARALD STEEN, GREGORY GRAZIANO, \\ ROBERT N. HENSINGER, ALAN SCHLESINGER, M. ANTHONY SCHORK, YU SHYR
}

From the University of Michigan, Ann Arbor, USA

The variability in measurement of angles in congenital scoliosis is not known, but it is postulated that it is larger than that in adolescent idiopathic scoliosis due to skeletal immaturity, incomplete ossification, and anomalous development of the end-vertebrae. To determine this variability, we selected 54 radiographs of adequate quality showing 67 scoliotic curves from children with congenital scoliosis. The end-vertebrae were preselected. Each curve was measured by the Cobb method on two separate occasions by six different observers, using the same goniometer and marker.

The intraobserver variability was $\pm 9.6^{\circ}$ and the interobserver variability $\pm 11.8^{\circ}$. If 'significant progression' is to be used as a criterion for surgical fusion in congenital scoliosis, there should be at least a $23^{\circ}$ increase, the entire range of the interobserver variability, in the curvature to ensure that the perceived increase is not due to variability in measurement.

J Bone Joint Surg [Br] 1995;77-B:768-70.

Received 12 March 1994; Accepted after revision 17 February 1995

Previous studies assessing variability of measurement in scoliosis (DeSmet et al 1982; Oda et al 1982; Goldberg et al 1988; Carman, Browne and Birch 1990; Morrissy et al

R. T. Loder, MD, Assistant Professor

A. Urquhart, MD. Senior Resident

G. Graziano, MD. Assistant Professor

R. N. Hensinger, MD, Professor

Section of Orthopaedic Surgery, University of Michigan Hospitals, 1500 East Medical Center Drive. 2912K TC, Box 0328, Ann Arbor, Michigan 48109-0328, USA.

H. Steen, MD, PhD, Orthopaedic Research Fellow

Biomechanics Laboratory. National Orthopaedic Center. Sophies Minde Orthopaedic Hospital, Trondheimsveien 132. Oslo N-0570. Norway.

A. Schlesinger, MD, Assistant Professor

Department of Radiology. Washington University School of Medicine, Mallinckrodt Institute of Radiology, 510 South Kingshighway, St Louis, Missouri 63110-1076, USA.

M. A. Schork, PhD. Professor

Y. Shyr, PhD, Instructor

Department of Biostatistics. School of Public Health, M4507 SPH II, Box 2029. University of Michigan. Ann Arbor, Michigan 48109. USA.

Correspondence should be sent to Dr R. T. Loder.

O1995 British Editorial Society of Bone and Joint Surgery

$0301-620 \mathrm{X} / 95 / 51046 \$ 2.00$
1990) have addressed only adolescent idiopathic scoliosis. There has been no study of congenital scoliosis in which operation is indicated when there is significant progression of the curve, regardless of age. Such progression must be greater than the normal variability of interobserver and intraobserver error in the radiological measurement of congenital scoliosis. Because of the vertebral anomalies in congenital scoliosis the end-vertebrae used in measuring the Cobb angle are less distinct, and therefore the interobserver and intraobserver error in measurement of this angle may be larger than that for adolescent idiopathic scoliosis.

Application of the values which are usually used to assess children with adolescent idiopathic scoliosis to those with congenital scoliosis may suggest the need for surgery when, in fact, significant progression has not occurred. We have therefore attempted to determine the variability in measurement of the Cobb angle in congenital scoliosis.

\section{PATIENTS AND METHODS}

We reviewed the posteroanterior spinal radiographs of 54 children with congenital scoliosis who were being followed at the University of Michigan Hospitals and Outpatient Clinics. Of these, 43 had one curve, nine two curves, and two three curves, giving a total of 67 curves. We included each child only once in the study since assessment of radiographs of the same child at two different ages was not allowed.

Each curve was measured twice by six different observers: three paediatric orthopaedic surgeons, one adult spine surgeon, one paediatric radiologist, and one mid-level resident in orthopaedic surgery. The end-vertebrae were preselected to reduce the component of variability (Morrissy et al 1990). Each observer drew endplate lines to determine the Cobb angle; these were erased after each reading. A minimum of three weeks was left between each set of measurements to reduce the effect of memory on the drawing of the endplate lines. The same goniometer (Zimmer Scoliosis Goniometer; Zimmer, Warsaw, Indiana) was used by all observers to eliminate the error between different goniometers (Morrissy et al 1990) as was the same, soft-lead marking pencil (black film marker, Dixon no. 2225; Joseph Dixon Company, Jersey City, New Jersey) for both sets of measurements.

We assessed both intraobserver and interobserver varia- 
bility using the methods described by Bland and Altman (1986). For intraobserver difference, the standard deviation is the square root of the residual mean square derived from a two-way ANOVA and included the curves by observer interaction with the 67 curves and six observers as main effects. The minimum difference required in two successive observations by the same observer based on $95 \%$ confidence is $\pm(\sqrt{\text { residual mean square }})(1.96)(\sqrt{2})$.

For interobserver variability we first averaged the two measurements of each observer and then performed a oneway ANOVA with the 67 curves as the main effect. From this ANOVA, the residual mean square $=$ between-observer variance + (within-observer variance/2). From the previous intraobserver analysis, we know the value for the withinobserver variance. We can thus derive that the betweenobserver variance $=$ the residual mean square (within-observer variance/2). The variance of a single measurement by a single observer equals the betweenobserver variance + the within-observer variance. The minimum difference between two observers in two successive observations based on $95 \%$ confidence is $\pm(\sqrt{\text { the variance }}$ of a single measure by a single observer $)(1.96)(\sqrt{2})$.

\section{RESULTS}

Intraobserver variability. The residual mean square from the two-way ANOVA for the measurement by observer interaction with measurements and observers as main effects was 11.99. Thus the intraobserver variability $=$ $(\sqrt{11.99})(1.96)(\sqrt{2})$, or $\pm 9.6^{\circ}$ (Fig. 1). There were no statistically significant differences in the mean intraobserver differences among the six different observers (Wilks lamb$\mathrm{da}=0.97$, MANOVA $\mathrm{F}=0.4, \mathrm{df}=5,62, \mathrm{p}=0.85$ ), indicating that the six observers were equally consistent in repeating measurements of the same radiograph.

Interobserver variability. There was a statistically significant difference among the six observers (Wilks lamb$\mathrm{da}=0.80$, MANOVA $\mathrm{F}=3.16, \mathrm{df}=5,62, \mathrm{p}=0.013$ ). Observers 2 and 3 measured the radiographs 'low' and observers 1, 4, 5 and 6 measured the radiographs 'high'.

The residual mean square from the one-way ANOVA with the radiographs as the main effect was 12.01 . Therefore, the between-observer variance $=12.01-(11.99) / 2$, or 6.01 . The variance of a single measure by a single observer $=11.99+6.01$, or 18.0. The minimum difference between two observers in two successive measurements $= \pm(\sqrt{18})(1.96)(\sqrt{2})$, or $\left.\pm 11.8^{\circ}\right)$.

\section{DISCUSSION}

To our knowledge this is the first study to assess the intraobserver and interobserver variability in the measurement of curves by the Cobb method in children with congenital scoliosis. All previous studies have involved children with adolescent idiopathic scoliosis.

The interobserver variability in children in our study was $\pm 11.8^{\circ}$, compared with values of $\pm 7.1^{\circ}$ (Ylikoski and Tallroth 1990) and $3.9^{\circ}$ (Carman et al 1990) obtained for adolescent idiopathic scoliosis. The intraobserver variability in our study was $\pm 9.6^{\circ}$, compared with $\pm 4.6^{\circ}$ (Ylikoski and Tallroth 1990) and $10.4^{\circ}$ (Carman et al 1990) for adolescent idiopathic scoliosis.

Only one study (Morrissy et al 1990) was controlled as rigorously as ours to reduce the non-observer causes of variability, such as preselection of the end-vertebrae, the use of the same goniometer, and the use of thin marking pencils instead of wax crayons, and it involved adolescent idiopathic scoliosis. Comparison of the two studies shows that both the intraobserver and interobserver errors are larger in children with congenital scoliosis than in idiopathic scoliosis. In addition, the $95 \%$ limits are nearly twice as large for congenital scoliosis than for idiopathic scoliosis as regards interobserver variability $\left( \pm 11.8^{\circ} v 6.3^{\circ}\right)$ and four times as large for intraobserver variability $\left( \pm 9.6^{\circ} v 2.8^{\circ}\right)$.

We did not assess the variability in the measurement of kyphosis. This is known to be slightly larger than that for scoliosis (Carman et al 1990) and we anticipate that the error in the measurement of congenital kyphosis is larger

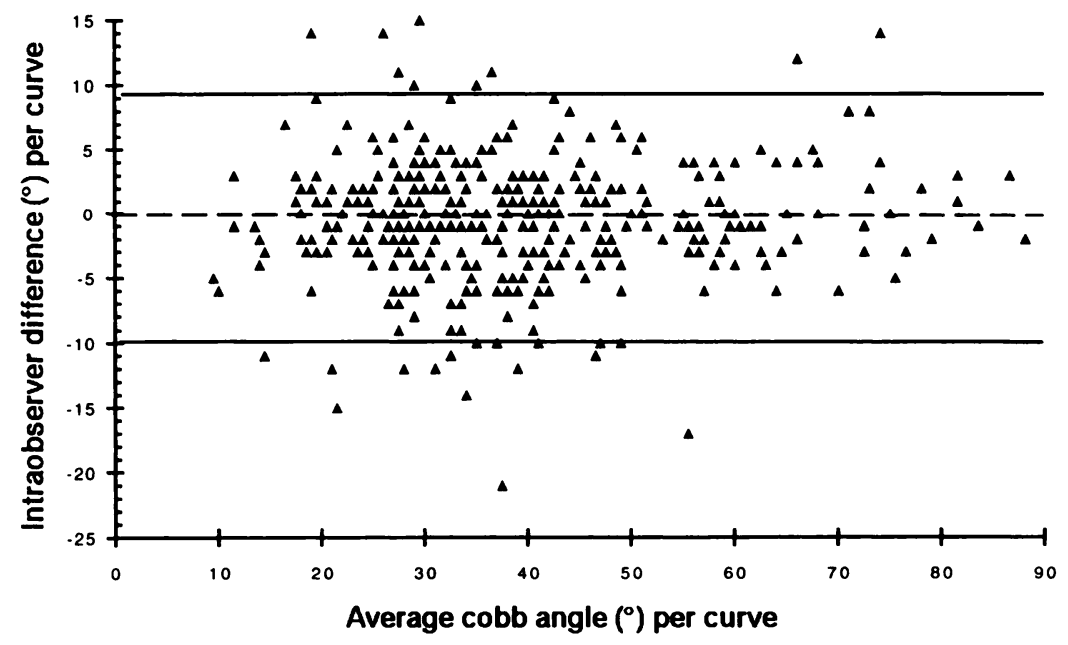

VOL. 77-B, No. 5, SEPTEMBER 1995
Fig. 1

A plot of the intraobserver difference between two successive measurements of the Cobb angle against the average Cobb angle. All 402 (67 scolioses times six different observers) differences are shown by the solid triangles. The hatched line gives the average intraobserver difference $\left(-0.3^{\circ}\right)$ and the solid lines are the $95 \%$ confidence limits for intraobserver difference. 
than the errors found in this study for congenital scoliosis.

One potential method of decreasing the error is by computer assistance (Jeffries et al 1980; Dutton et al 1989). Dutton et al (1989) noted a decrease in intraobserver error from $4.3^{\circ}$ to $1.5^{\circ}$ and a decrease in interobserver error from $4.9^{\circ}$ to $1.6^{\circ}$ when using computers. Jeffries et al (1980) found a decrease in the $95 \%$ tolerance limits for intraobserver error from $6.6^{\circ}$ to $2.8^{\circ}$ and from $11.1^{\circ}$ to $3.3^{\circ}$ for interobserver error. Both methods depend on digitising many data points from each of the vertebrae, creating quantitative models of the entire spinal pattern, and then mathematically manipulating the resultant computer-generated curves (first and second calculus derivatives) to determine the Cobb angle. We believe that the main reason for the large variability in our study was the more variable and irregular end-vertebrae in children with congenital scoliosis. It is not known whether digitisation and computer-assisted analysis of congenital scoliosis would decrease this source of error, but we suspect that there will still be large errors due to the inherent problems of selecting the points for digitisation on these anomalous, irregular vertebrae.

If the Cobb method is used to determine progression of a curve in congenital scoliosis between two measurements, and based on two different observers at different times, then at least $23^{\circ}$ of change is necessary to ensure with $95 \%$ confidence that the increase in the curve is not due to chance or error of measurement but to an increase in the curve itself. This margin of error may be reduced to $19^{\circ}$ if the same observer performs both measurements. These margins of error in measurement are larger than those in adolescent idiopathic scoliosis and question this method of measurement for children with congenital scoliosis. More accurate and precise methods of assessment are needed.

This study was funded by the Fischer Endowment for Pediatric Orthopaedic Research. University of Michigan. Statistical support was provided by the University of Michigan Multipurpose Arthritis and Musculoskeletal Diseases Center, NIH grant no. 2P60AR20557.

No benefits in any form have been received or will be received from a commercial party related directly or indirectly to the subject of this article.

\section{REFERENCES}

Bland JM, Altman DG. Statistical methods for assessing agreement between two methods of clinical measurement. Lancet 1986;i:307-10.

Carman DL, Browne RH, Birch JG. Measurement of scoliosis and kyphosis radiographs, intraobserver and interobserver variation. $J$ Bone Joint Surg $[A m]$ 1990:72-A:328-33.

DeSmet AA, Goin JE, Asher MA, Scheuch HG. A clinical study of the differences between the scoliotic angles measured on posteroanterior and anteroposterior radiographs. $J$ Bone Joint Surg [Am] 1982:64-A:489-93.

Dutton KE, Jones TJ, Slinger BS, O'Connor J. Reliability of the Cobb angle index derived by traditional and computer assisted methods. Australas Phys Eng Sci Med 1989:12:16-23.

Goldberg MS, Poitras B, Mayo NE, et al. Observer variation in assessing spinal curvature and skeletal development in adolescent idiopathic scoliosis. Spine 1988:13:1371-7.

Jeffries BF, Tarlton M, DeSmet AA, Dwyer SJ III, Brower AC. Computerized measurement and analysis of scoliosis: a more accurate representation of the shape of the curve. Radiology 1980;134:381-5.

Morrissy RT, Goldsmith GS, Hall EC, Kehl D, Cowie GH. Measurement of the Cobb Angle on radiographs of patients who have scoliosis: evaluation of intrinsic error. J Bone Joint Surg [Am] 1990:72-A:320-7.

Oda M, Rauh S, Gregory PB, Silverman FN, Bleck EE. The significance of roentgenographic measurement in scoliosis. $J$ Pediatr Orthop 1982;2:378-82.

Ylikoski M, Tallroth $\mathbf{K}$. Measurement variations in scoliotic angle. vertebral rotation, vertebral body height, and intervertebral disc space height. J Spinal Disord 1990;3:387-91. 